\title{
Automated Contactless Temperature and Facemask Detection Using Deep Learning
}

\author{
Pratik A. Malave ${ }^{1}$, Sachin M. Wagde르, Iresh S. Vacche ${ }^{1}$, Manali S. Gaikwad ${ }^{1}$, Bapuraje Arkas ${ }^{2}$ \\ ${ }^{1}$ Department of Computer Engineering, Shree Ramchandra College of Engineering, Pune, Maharashtra, India \\ ${ }^{2}$ Assistant Professor, Department of Computer Engineering, Shree Ramchandra College of Engineering, Pune, \\ Maharashtra, India
}

\begin{abstract}
Article Info

Volume 8, Issue 3

Page Number : 521-527

Publication Issue

May-June-2021

Article History

Accepted : 01 June 2021

Published : 06 June 2021

In Our proposed project we are using raspberry pi to detect face mask and temperature of a person. COVID-19 is an infectious disease caused by the corona virus. Corona virus is nothing but a family of viruses which cause the illness in humans. The common symptoms of this virus are fever, dry cough, breathing problem, etc. It is necessary to maintain a social distance and wear a face mask to avoid the chances of getting the virus, as it is affecting the whole world. And for this we need a system which will keep an eye on everyone to ensure the safety of ourselves as well as of others. To ensure the safety of the public, we tried to build a system for contactless Face Mask and Temperature Detection.

This project will be helpful in crowded places or workstations to provide support for the prevention and control of Covid-19.
\end{abstract}

Keywords - Deep Learning, TensorFlow, OpenCV, Dlib

\section{INTRODUCTION}

A new strain that has not previously been identified in humans is a novel coronavirus. Corona viruses are a wide group of viruses which cause illness that ranges from colds to deadly infections like Middle East Respiratory Syndrome (MERS) and Severe Acute Respiratory Syndrome (SARS). COVID-19 has become a pandemic all over the world. People all over the globe face challenging situations because of this pandemic. Every day many people are being infected and died. The numbers are increasing day by day.
Fever, dry cough, tiredness, diarrhea, loss of taste, and smell are the major symptoms of coronavirus which is declared by the World Health Organization (WHO). Many precautionary measures are taken to fight against coronavirus. Among them cleaning hands, maintaining social distancing, wearing a mask, using sanitizers.

The pandemic situation has taken over the world and has made the conditions worst, as of now there are some vaccines developed for the contagious disease and hence wearing masks and maintain social 
distancing has become some of the efficient methods to stop the spread of COVID-19. The cases have been escalating at a very fast rate all over the world and thus wearing masks is important. To monitor masks at public places, this survey paper provides a solution. In this pandemic period using CCTV, we can keep track of human activities in public places, and henceforth we are able to compute and summarize whether someone is wearing a mask or not. This proposed survey will also restrict people from coming together and prevent social gatherings. People who gather in massive amounts at religious places can make conditions worse. Recently most of the countries within the world are in the lockdown period and this has imposed the citizens to be at home but as time passes people will tend to go to more and more public places and religious places, so in those circumstances this method of monitoring masks will be beneficial for all around the world. With the help of deep learning and the installed CCTV, we can keep track of humans and compute whether a person is wearing a mask or not. We are also measuring the body temperature of a person, which can help reduce the spread of COVID19.

Face mask detection is a technique to seek out whether someone is wearing a mask or not. It is like detecting an object from a scene. Many systems have been introduced for object detection. Deep learning techniques are highly used in medical applications. Recently, deep learning architectures have shown a remarkable role in object detection. These architectures can be incorporated in detecting the mask on a face. The trend of wearing face masks in public is rising due to the COVID- 19 corona virus epidemic all over the world. Before Covid-19, People used to wear masks to protect their health from air pollution. While other people are self-conscious about their looks, they hide their emotions from the public by hiding their faces. Scientists proofed that wearing face masks works on impeding COVID-19 transmission. COVID-19 (known as corona virus) is the latest epidemic virus that hit the human health in the last century. In 2020, the rapid spreading of COVID-19 has forced the World Health Organization to declare COVID- 19 as a global pandemic. The virus spreads through close contact and in crowded and overcrowded areas. The corona virus epidemic has given rise to an extraordinary degree of worldwide scientific cooperation. Artificial Intelligence (AI) based on Machine learning and Deep Learning can help to fight Covid-19 in many ways. Machine learning allows researchers and clinicians evaluate vast quantities of data to forecast the distribution of COVID-19, to serve as an early warning mechanism for potential pandemics, and to classify vulnerable populations. The provision of healthcare needs funding for emerging technology such as artificial intelligence, IoT, big data and machine learning to tackle and predict new diseases. To better understand infection rates and to trace and quickly detect infections, the AI's power is being exploited to address the Covid-19 pandemic. People are forced by laws to wear face masks in public in many countries and check their body temperature thrice a day. These rules and laws were developed as an action to the exponential growth in cases and deaths in many areas. The monitoring process involves the detection of anyone who is not wearing a face mask. Here we introduce a contactless temperature and face mask detection model that is based on computer vision and deep learning. The proposed model is integrated with IOT to impede the COVID-19 transmission by allowing the detection of people who are wearing masks not wearing face masks and having body temperature $\leq 37^{\circ} \mathrm{C}$. The model is integration between deep learning and classical machine learning techniques with OpenCV, TensorFlow, keras and IOT frameworks. We have used deep transfer leering for feature extractions and combined it with three classical machine learning algorithms. We introduced a comparison between them to find the most suitable algorithm that achieved the highest accuracy and 
consumed the least time in the process of training and detection.

\section{RELATED WORK}

Mohammad Marufur Rahman, Md. Motaleb Hossen Manik, Md. Milon Islam, Saifuddin Mahmud and Jong-Hoon Kim proposed a system to find out whether a person is using a mask or not and informing the corresponding authority in a smart city network. Firstly, CCTV cameras are used to capture real-time video footage of different public places in the city. From that video footage, facial images are extracted and these images are used to identify the mask on the face. The learning algorithm Convolutional Neural Network (CNN) is used for feature extraction from the images then these features are learned by multiple hidden layers.Vinitha and Velantina proposed a mask face detection model that is basedon computer vision and deep learning. There proposed model is integrated with surveillance cameras to impede the COVID-19 transmission by allowing the detection of people who are wearing masks not wearing face masks. There model is integration between deep learning and classical machine learning techniques with OpenCV, tensor flow and keras. They have used deep transfer leering for feature extractions and combined it with three classical machine learning algorithms.They introduced a comparison between them to find the most suitable algorithm that achieved the highest accuracy and consumed the least time in the process of training and detection.

Md. Rafiuzzaman Bhuiyan, Sharun Akter Khushbu and Md. Sanzidul Islam proposed a system in which theydivided their work into two parts. Firstpart they are discussing about data acquisition and annotationpart. Then they briefly cover about our dataset also preprocessing stuff. Next they used data annotation.

Isack Farady, Chih-Yang Lin, Amornthep Rojanasarit , Kanatip Prompol and Fityanul Akhyar proposed a system in which theyused deep learning to detect whether someone is wearing a mask properly or not. First, they build a network to learn the features of there input image dataset to recognize three kinds of mask- wearing positions. They define those positions into three classes of the object, then set the classification sub-network and regression subnetwork in the last layer of the RetinaNet. The output of Module 1 and Module 2 determine the class of a mask-wearing position and show the bounding box on the predicted class. In Module 3 they combine two predicted results from Modules 1 and 2 then generate an image with a bounding box and show the highest temperature of a predicted head bounding box from the thermal camera.

\section{1) DATA SET-}

Data is really important in data driven techniques like machine learning, deep learning. The more the data the more the better result. For our purpose of working with Tenserflow we also need more data and with proper annotate. But for our work we don't find any type of annotate data. Using webscrapping tool from website we have collected 1394 images of both mask and no-mask.Next our data is not suitable for fed into the model. Before feeding we do some pre-processing.There are some irrelevant images inside the dataset. We remove them \& fifinally got our dataset with 1376 images where 690 images for mask and 686 for no- mask. Fig-1 shows the sample of our dataset.
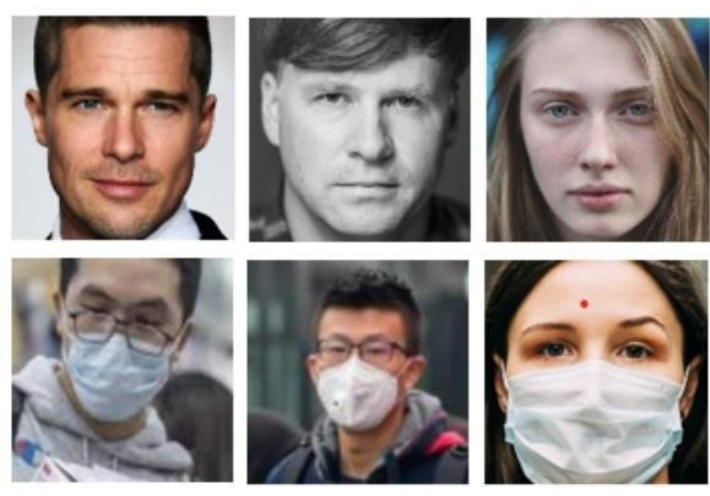

Fig-1.Dataset 


\section{2) PROPOSED SYSTEM-}

As countries around the globe are reopening their doors, living with the corona virus is becoming the new manner of existance, however to prevent the spread of Covid-19, we need to split people having the corona virus from relaxation.Social distancing

, wearing a face mask and having body temperature $<37^{\circ} \mathrm{C}$ these are the three most recommended precautions by WHO to the nations around the world for safety of peoples from Covid-19.

According to WHO wearing a face mask in public places gets lowers chance of being inflacted by Covid19.According to a report of CDC, fever is the leading symptom of corona virus with up to $83 \%$ of symptomatic patients are showing some signs of fever.Many countries are making temperature checkups and mask mandatory for schools, colleges, offices and other workplaces.

Currently temperature checkups are done manually using contactless thermometer.Manual checkups can be inefficient, impractical(Crowdy Places) and risky.To solve these problem we have build a device thet automates the process of temperature checkup by using facial landmarking \& contactless IR temperature sensor and mask detection using deep learning neural network.Fig-2.showing the basic architecture of proposed system.

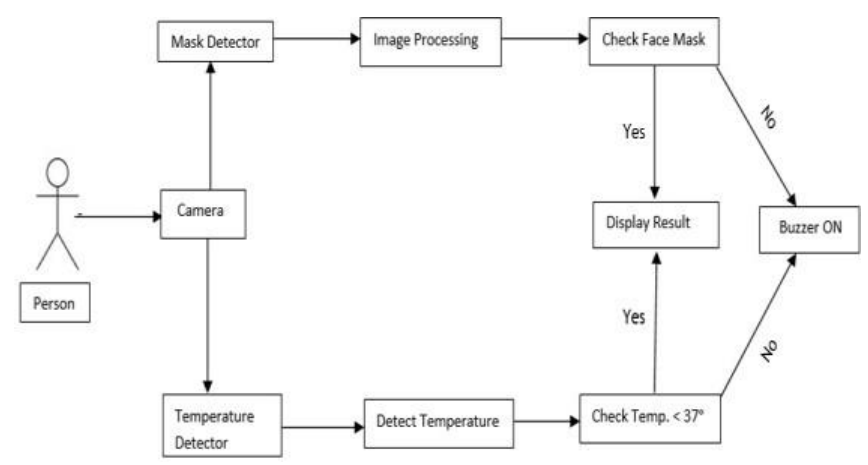

Fig.2:-Proposed System Diagram

The use of this device is not limited to Schools, Colleges, Offices but it can also be use in high risk areas like Bus Stop, Railway Station, Movie Theaters, Hospitals, etc.In this device the camera are used to capture image and infrared temperature sensor for temperature checkup.The image is capture by the camera is feed into a system that identifies, if the person ia wearing face mask or not, if the person is wearing face mask and having body temperature $<37^{\circ} \mathrm{C}$ then entry is allowed for that person or If the person without a face mask appears in the image or having body temperature $>37^{\circ} \mathrm{C}$ is detected then a alert is generated by the buzzer for the certain authority to take necessary actions.

The proposed system focuses on how to identify the person on image is wearing face mask and temperature with the help of computer vision and deep learning algorithm by using the OpenCV, Tensor flow, Keras and DLIB library and infrared sensor.

Data at Source:-The majority ofthe images were augmented by OpenCV. The set of images were already labeled "mask" and "no mask". The images that were present were of different sizes andresolutions, probably extracted from different sources or from machines (cameras) of different resolutions.

\section{3) Data Preprocessing:-}

Data preprocessing involves conversion of data from a given format to much more user friendly, desired and meaningful format. It can be in any form like tables, images, videos, graphs, etc. These organized information fifit in with an information model or composition and captures relationship between different entities. The proposed method deals with image data using Numpy and OpenCV.

\section{4) Algorithms:}

\section{ALGORITHM 1:FACE MASK DETECTION}

INPUT:Datasetincluding faces with and without mask OUTPUT:Catrgorized image depicting the presence of face mask. 
For each image the dataset do

- Visualize the image in two categories (with and without images)and lable them

- Convert RGB-image into GRAY- Scale image

- Resize the GRAY-SCALE image into $254^{*} 254$

- Normalize the image and expand the dimentions of the array.

End loop

Spit the data and train the model

For each image check the mask probability

If mask_probability $>0.5$ then

Return true

Else

Return False

\section{ALGORITHM 2:TEMPERATURE DETECTION}

Temperature $\mathrm{T}=0$

Detect the face landmark points using DLIB.

Select the best points on forehead to take temperature.

Take the temperature from selected points using Infrared Sensor.

If Temperature $<37^{\circ} \mathrm{C}$ then

\section{Return True}

Else

\section{Return False}

\section{5) INCORPORATED PACKAGES}

\section{A. TensorFlow}

TensorFlow is an interface for expressing machine learning algorithms, is utilized for implementing $\mathrm{ML}$ systems into fabrication over a bunch of areas of computer science, including sentiment analysis, voice recognition, geographic information extraction, computer vision, text summarization, information retrieval, computational drug discovery and flaw detection to pursue research.

\section{B. Keras}

Keras gives fundamental reflections and building units for creation and transportation of ML arrangements with high iteration velocity. It takes full advantage of the scalability and cross-platform capabilities of TensorFlow. The core data structures of Keras are layers and models.It helps to conversion of the class vector to the binary class matrix in data processing, it helps to compile the overall model.

\section{OpenCV}

OpenCV (Open Source Computer Vision Library), an open source computer vision and ML software library, is utilized to differentiate and recognize faces, recognize objects, group movements in recordings, trace progressive modules, follow eye gesture, track camera actions, expel red eyes from pictures taken utilizing flash, find comparative pictures from an image database, perceive landscape and set up markers to overlay it with increased reality and so forth. The proposed method makes use of these features of OpenCV in resizing and color conversion of data images

\section{DLIB}

Dlib is an open source general purpose software library implemented in $\mathrm{C}++$ and contains software components spanning various domains. Other off the shelf implementations of facial landmark detection are available in the openCV library, which is a library of components dedicated to computer vision. However, the dlib implementation was used in this study because it has a higher accuracy of facial landmark detection compared to the implementations available in the openCV library.

\section{Conclusion}

In our study, we have introduced an approach for detecting a person is wearing a mask or no mask. It'll perform really well in images and our detection results was also quite good. Later we also applied this model into a real-time video to check whether our model's fps rate inside video and its detection performance with two classes mask/no mask. Inside video our model get impressive output where average fps is 17 . The paper presents a system for a 
smart city to reduce the spread coronavirus by informing the authority about the person is wearing a facial mask that is precautionary measure of Covid19. The motive of the work comes from the people disobeying the principles that are mandatory to prevent the spread of coronavirus. Though the dataset that we collected is not that much variety after all it gives us promising accuracy in testing with some real-world data. We used OpenCV, tensor flow and DLIB to detect whether people were wearing face masks or not. The models were tested with images and real-time video streams. The accuracy of the model is achieved and, the optimization of the model may be a continuous process and that we are building a highly accurate solution by tuning the hyper parameters. This specific model might be used as a use case for edge analytics. Furthermore, the proposed method achieves state-of- the-art results on a public face mask dataset. By the event of face mask detection, we will detect if the person is wearing a mask and permit their entry would be of great help to the society. The system contains a mask detection architecture where a deep learning algorithm is employed to detect the mask on the face. To train the model, labelled image data are used where the pictures were facial images with masks and without a mask. The deployed model will contribute immensely to the public health care system. In future it can be extended to detect if a person is wearing the mask properly or not. The model can be further improved to detect if the mask is virus prone or not i.e. the type of the mask is surgical, N95 or not.

In this paper, we briefly explained the motivation of the work at first. Then, we illustrated the learning and performance task of the model. Using basic ML tools and simplified techniques the method has achieved reasonably high accuracy. It are often used for a spread of applications. Wearing a mask may be obligatory in the near future, considering the Covid-
19 crisis. Many public service providers will ask the customers to wear masks correctly to avail of their services.

\section{REFERENCES}

[1]. Swann OV, Holden KA, Turtle L, et al. Clinical characteristics of children and young people admitted to hospital with covid-19 in United Kingdom: prospective multicentre observational cohort study. BMJ. 2020 Aug 27;370:m3249.

[2]. Mehta NS, Myton OT, Mullins EWS, et al. SARS-CoV-2 (COVID-19): what do we know about children? A systematic review. Clin Infect Dis. 2020 May 11 [Epub ahead of print].

[3]. Castagnoli R, Votto M, Licari A, et al. Severe acute respiratory syndrome coronavirus 2 (SARS-CoV-2) infection in children and adolescents: a systematic review. JAMA Pediatr. 2020 Sep 1;174(9):882-9.

[4]. Centre for Disease Control and Prevention. COVID-19 pandemic planning scenarios. 2020 [internet publication].

[5]. World Health Organization. Public health surveillance for COVID-19: interim guidance. 2020 [internet publication].

[6]. Choi GJ, Kim HM, Kang H. The potential role of dyslipidemia in COVID-19 severity: an umbrella review of systematic reviews. J Lipid Atheroscler. 2020 Sep;9(3):435-48.

[7]. https://www.google.com/amp/s/data-

flair.training/blogs/face-mask-detectionwithpython/amp/

[8]. https://www.irjet.net > archivesPDF

[9]. Covid-19 Facemask Detection with Deep Learning and Computer Vision - IRJET

[10]. "Coronavirus Disease 2019 (COVID-19) Symptoms", Centers for Disease Control and Prevention, 2020. [Online]. Available: https://www.cdc.gov/coronavirus/2019ncov/sy mpto mstesting/symptoms.html. 2020. 
[11]. "Coronavirus - Human Coronavirus Types CDC", Cdc.gov, 2020. [Online].Available: https://www.cdc.gov/coronavirus/types.html. 2020.

[12]. W.H.O., "Advice on the use of masks in the context of COVID-19: interim guidance", 2020.

[13]. M. Jiang, X. Fan and H. Yan, "RetinaMask: A Face Mask detector”, arXiv.org, 2020. [Online]. Available: https://arxiv.org/abs/2005.03950. 2020.

\section{Cite this article as :}

Pratik A. Malave, Sachin M. Wagde, Iresh S. Vacche, Manali S. Gaikwad, Bapuraje Arkas, " Automated Contactless Temperature and Facemask Detection Using Deep Learning", International Journal of Scientific Research in Science and Technology(IJSRST), Print ISSN : 2395-6011, Online ISSN : 2395-602X,Volume 8, Issue 3, pp.521-527, May-June-2021. Available at doi $\quad$ : https://doi.org/10.32628/IJSRST2183116 Journal URL : https://ijsrst.com/IJSRST2183116 